\title{
REMARKS ON "THE CLASSIFICATION OF REVERSIBLE CUBIC SYSTEMS WITH CENTER"
}

\author{
HENRYK ŻOŁĄDEK
}

The paper [8] from the title was very long and rather technical. Nevertheless it has drawn attention of some people who have pointed out to the author some mistakes and inaccuracies. Especially, the author thanks A. P. Sadovski for sending him a list of mistakes. Also the author himself found some misprints, mistakes and one incomplete proof. Moreover, some new results about this subject have appeared meantime.

\section{The problem of center}

In the introduction of [8] four classes of polynomial vector fields with center were introduced: with Darboux integral, with Darboux-Schwartz-Christoffel (DSC) integral, with Darboux-hyperelliptic (DHE) integral, and rationally reversible ones.

The Darboux integrals are of the form

$$
H=e^{g} \prod f_{j}^{a_{j}}
$$

where $f_{i}(x, y)$ are polynomials and $g(x, y)$ is a rational function.

The DSC integrals are of the form

$$
H=W e^{g(U)} \prod\left(U-u_{j}\right)^{a_{j}}+\int^{U} e^{g(u)} \prod\left(u-u_{j}\right)^{a_{j}-1} P(u) d u
$$

1991 Mathematics Subject Classification. 34C05, 58F21.

Supported by Polish KBN Grant No 2 P03A 02208. 
where $U(x, y), W(x, y), g(\cdot)$ are rational functions and $P(\cdot)$ is a polynomial.

The DHE integrals are of the form

$$
H=\prod\left(\frac{R_{i}-\sqrt{S(X)}}{R_{i}+\sqrt{S(X)}}\right)^{a_{i}} \exp \left[T \sqrt{S(X)}+\int^{X} W(u) \sqrt{S(u)} d u\right]
$$

with rational functions $R_{i}(x, y), X(x, y), T(x, y)$ and $X(\cdot), W(\cdot)$. A rationally reversible system $V$ at a center $O$ admits some rational non-invertible map $\Phi$ : $\mathbb{R}^{2} \rightarrow \mathbb{R}^{2}$ and a polynomial vector field $V^{\prime}$ on $\mathbb{R}^{2}$ such that: (i) $\Phi_{*} V$ and $V^{\prime} \circ \Phi$ are collinear; (ii) the curve of non-invertibility $\Gamma_{\Phi}$ of $\Phi$ passes through $O$ and there is a neighbourhood $\mathcal{U} \subset \mathbb{R}^{2}$ of $O$ such that the boundary of $\Phi(\mathcal{U})$ contains a part the curve $\Gamma^{\prime}=\Phi\left(\Gamma_{\Phi}\right)$, the vector field $V^{\prime}$ is tangent to $\Gamma^{\prime}$ at $\Phi(O)$ from the outside of $\Phi(\mathcal{U})$ and $V^{\prime}(\Phi(O)) \neq 0$. (As noticed by D. Schlomiuk, in [8] the author has erroneously written that $V^{\prime}$ is a polynomial vector field on $\mathbb{R} P^{2}$.) Now the author thinks that one should consider another classification of known cases of centers for polynomial vector fields: with Liouvillian integral (see below) and rationally reversible.

However, in [2] it was proved that there exist local analytic vector fields with center which are not reversible (by means of a local analytic non-invertible map) and are not locally Liouvillian integrable (where in the definition below one starts from the field of germs of meromorphic functions). These centers are non-elementary. Thus one should not expect that non-elementary centers can be easily classified. One may hope that the alternative: either rationally reversible or Liouvillian integrable, holds for polynomial elementary centers and for polynomial nilpotent centers. Also the author's conjecture that any cubic center is either rationally reversible or Darboux integrable remains unchanged.

The class of Liouvillian functions (or functions expressed by quadratures) on $\mathbb{C}^{n}$ is defined as the class obtained from rational functions by application of the operations: algebraic operations, compositions, exponentiations $\left(e^{f(x)}\right)$ and integration $\left(\int^{x} f d x_{i}\right)$. Liouvillian functions include Darboux integrals, DSC integrals and DHE integrals. Recently there have appeared two important papers about Liouvillian functions.

In [6] Singer has shown (using differential algebra) that if a polynomial vector field on $\mathbb{C}^{2}$ has a Liouvillian first integral then the integrating factor can be chosen in the form $M=\exp \left(\int^{(x, y)} U d x+V d y\right)$, where $U d x+V d y$ is a closed rational 1-form. Christopher [4] has noticed that this multiplier can be written in the Darboux form $M=e^{g} \prod f_{i}^{a_{i}}$.

In [5] Khovanski has published the proofs of half of his Ph.D. thesis (written 25 years ago). There he introduces the monodromy group of a function (its definition is also in [8]). This group is a topological invariant. Functions expressible 
by quadratures have solvable monodromy groups. In particular, the monodromy group associated with the Darboux integral is abelian.

Recently, the author has found a large class of Liouvillian first integrals which he calls multiple DSC integrals:

$$
H=Y S(X)^{p / q}+\int^{X} s(u)^{p / q} W(u) d u+\sum_{i=1}^{s} \int^{X_{i}} u^{p / q} \prod_{j=1}^{r_{i}}\left(u-u_{i j}\right)^{a_{i j}} d u
$$

where $X(x, y), Y(x, y), X_{i}(x, y), S(\cdot), W(\cdot)$ are rational functions, $p / q, a_{i j}$ are rational numbers and $u_{i j} \in \mathbb{C}$. Some additional conditions must be satisfied in order that these functions represent first integrals for polynomial vector fields.

ExAmpLES. 1. The logarithm of the DHE integral is

$$
T \sqrt{S(X)}+\int^{X} \sqrt{S(u)} W(u) d u+\sum 2 a_{i} \int^{S(X) / R_{i}^{2}} \frac{\sqrt{u} d u}{1-u} .
$$

2. The system

$$
\begin{aligned}
& \dot{x}=-y\left(1+x^{n}+\lambda y^{n}-2 \lambda x^{2} y^{n-2}\right)=P(x, y), \\
& \dot{y}=x\left(1+x^{n}+\lambda y^{n}-2 x^{2} y^{n-2}\right)=Q(x, y),
\end{aligned}
$$

has a first integral

$$
\begin{aligned}
H= & \int f^{-(n+4) /(2 n)}(Q d x-P d y) \\
= & \frac{(4 \lambda)^{2 / n}}{n} \int^{X_{1}} u^{-(n+4) /(2 n)}(u-1)^{(2-n) / n} d u \\
& +\frac{4^{2 / n}}{n} \int^{X_{2}} u^{-(n+4) /(2 n)}(u-1)^{(2-n) / n} d u
\end{aligned}
$$

where

$$
\begin{aligned}
f & =1+2\left(x^{n}+\lambda y^{n}\right)+\left(x^{n}-\lambda y^{n}\right)^{2}, \\
X_{1} & =f\left(1-x^{n}+\lambda y^{n}\right)^{-2}, \quad X_{2}=f\left(1+x^{n}-\lambda y^{n}\right)^{-2} .
\end{aligned}
$$

This example comes from the work [3] of Chavarriga, where the existence of the integrating factor was proved.

The monodromy groups associated with DSC integrals and with multiple DSC integrals are solvable and can be embedded into the group of affine diffeomorphisms of a complex line. Using the Singer theorem one can prove that the same holds for any Liouvillian first integral. Now the author is working on the proof that any Liouvillian first integral is of the Darboux, DSC or multiple DSC type. 


\section{Rational curves in $\mathbb{C} P^{2}$}

In the proof of Proposition 1 (p. 116 of [8]) the author needed the following fact: If $C \subset \mathbb{C}^{2}$ is a rational curve then there is an invertible rational map $(x, y) \rightarrow(X, Y)$ such that $C=\{X=0\}$. It is the Abhyankar-Moch theorem [1], well known in algebraic geometry. The author thanks T. Maszczyk for this reference. The very short arguments given in [8] are insufficient.

\section{Cubic centers}

In Theorem 1 of [8] the list of reversible centers is presented. There are given only the maps $\Phi$ and the vector fields $V^{\prime}$ (in the image). The formulas for the vector fields $V$ are given in the course of the proofs in Section 5. However, not all formulas are there, some are in implicit form and (as Sadovski has pointed out) they contain some misprints and mistakes. Sadovski investigated the center conditions using different methods (reduction to a system of non-linear oscillations and applying the Cherkas methods). He informed the author that several of the cases from [8] coincide with some of his cases. Because there is an interest we present a complete list of reversible $V$ 's.

The author together with J. Sokulski tried to classify the cubic Darboux centers. We have found 35 cases but we do not know whether the list is complete. Sokulski [7] gave a complete list but under some restrictions (on degrees etc.). We use the oportunity to present also all known cubic Darboux centers, where the first 18 cases come from [7] and the others are new. Thus we have all 52 cases of cubic centers in one place.

Reversible centers (The upper index denotes the codimension).

$$
\begin{array}{ll}
C R_{1}^{(7)}: & \dot{x}=k+l x^{2}+m y+n y^{2}+p x^{2} y+q y^{3} \\
& \dot{y}=2 x\left(r+s x^{2}+t y+q y^{2}\right) \\
C R_{2}^{10)}: \quad & \dot{x}=\left(k+l x+m x^{2}\right)(2 x+y) \\
& \dot{y}=y\left[(n+p x)(x+y)+q y^{2}+\left(k+l x+m x^{2}\right)\right] \\
C R_{3}^{(10)}: \quad & \dot{x}=\left(k+l x+m x^{2}\right) y \\
& \dot{y}=A+B x+C x^{2}+D x y+E y^{2}+F x^{3}+G x^{2} y+H x y^{2}+I y^{3}
\end{array}
$$

where $A=2\left[\left(3 a-b^{2}\right) b k+\left(b^{2}-2 a\right) l-b m\right], B=2\left[\left(2 a^{2}+2 a b^{2}-b^{4}\right) k+\left(b^{2}-a\right) b l-\right.$ $\left.\left(2 a+b^{2}\right) m\right], C=2 a\left[\left(5 a-2 b^{2}\right) b k+2\left(b^{2}-a\right) l-3 b m\right], D=3 A / 2, E=2 b k-l$, $F=2 a^{2}\left[\left(2 a^{2}-b\right) k+b l-2 m\right], G=3 F /(2 a), H=\left(5 a / 2-b^{2}\right) k+b l-m, I=k / 2$. This case is parametrized by $a, b, c, k, l, m$. (In Theorem 1 of [8] the formula for $p$ should be changed to $p=4 a\left[\left(2 a-b^{2}\right) k+b l-2 m\right]$.)

$$
\begin{array}{ll}
C R_{4}^{(8)}: & \dot{x}=-(x+c)(k x y+l+m T x)-x\left(n y^{2}+p T y+q T^{2}\right) \\
& \dot{y}=(2 x+y+c)\left(n y^{2}+p T y+q T^{2}\right)-y(k x y+l+m T x)
\end{array}
$$


Here and in the cases $C R_{i}^{(*)}, i \neq 16$, below,

$$
T=x+y+c .
$$

$$
\begin{aligned}
& C R_{5}^{(8)}: \quad \dot{x}=-k x y^{2}-l+m x^{2} y-(n x y+p+q T x)(2 x+y+c) \\
& \dot{y}=x\left[l+p+m c x+(k+n) x y+m x^{2}+q T x\right] \\
& C R_{6}^{(7)}: \quad \dot{x}=-n T+n y+(l-k-p) x y^{2}+(m-l-q) x y T-(m+r) x T^{2} \\
& \dot{y}=-n y+(p-l) x y^{2}+k y^{3}+(q-m) x y T+p y^{2} T+r x T^{2} \\
& +q y T^{2}+r T^{3} \\
& C R_{7}^{(9)}: \quad \dot{x}=x[-(n+k)+(l-m) x y-(l+p) x T] \\
& \dot{y}=n x+k y+n T+(m-l) x^{2} y+p x^{2} T+m x y T+p x T^{2} \\
& C R_{8}^{(10)}: \quad \dot{x}=x[-k-2 n T+2(l-m) x y-l x T] \\
& \dot{y}=2\left[k y+n x T+n T^{2}+(m-l) x^{2} y+m x y T\right] \\
& C R_{9}^{(10)}: \quad \dot{x}=x\left[-l T+2(l-m) y-k x y-2 n T^{2}\right] \\
& \dot{y}=2\left[(m-l) x y+m y T+k x y^{2}+n x T^{2}+n T^{3}\right] \\
& C R_{10}^{(10)}: \quad \dot{x}=x\left[-k+3(l-m) x y-l x T-3 n T^{2}\right] \\
& \dot{y}=3\left[k y+n x T^{2}+(m-l) x^{2} y+m x y T+n T^{3}\right] \\
& C R_{11}^{(7)}: \quad \dot{x}=2 x\left[-(p+k) x+(l-n) y^{2}+(m-l-q) y T-(m+r) T^{2}\right] \\
& \dot{y}=2 p x^{2}-k x y-p x T+2(n-l) x y^{2} \\
& +2(q-m) x y T-n y^{2} T+2 r x T^{2}-q y T^{2}-r T^{3} \\
& C R_{12}^{(7)}: \quad \dot{x}=2\left[(m-k-q) x y-(m+r) x T+l y^{3}+(p-n) y T^{2}\right. \\
& \left.+(n-l) y^{2} T-p T^{3}\right] \\
& \dot{y}=-k y^{2}+2(q-m) x y+2 r x T-2 l y^{3}-p q T \\
& -r T^{2}-2 n y^{2} T-2 p y T^{2} \\
& C R_{13}^{(10)}: \quad \dot{x}=3 x\left[-k x+2(l-m) y^{2}-l y T-2 n x T\right] \\
& \dot{y}=2\left[-k x y-m y^{2} T+3(m-l) x y^{2}+3 n x^{2} T-n x T^{2}\right] \\
& C R_{14}^{(9)}: \quad \dot{x}=3\left[-k x y-2 p x^{2}+2 l y^{3}+2(m-n) x y T-l y^{2} T-m x T^{2}\right] \\
& \dot{y}=2\left[3 p x^{2}-k y^{2}-p x T-3 l y^{3}+3(n-m) x y T-n y T^{2}\right] \\
& C R_{15}^{(10)}: \quad \dot{x}=(2 y-T)\left(k y^{2}+l x\right)-4 x\left(m y+n T^{2}\right) \\
& \dot{y}=(4 x-T)\left(m y+n T^{2}\right)-2 y\left(k y^{2}+l x\right) \\
& C R_{16}^{(5)}: \quad \dot{x}=-x(k y+l x)-(m y+n x) T_{2} \\
& +(b x+2 c y+e)\left[-q x^{2}+(n-p) x y+m y^{2}\right] \\
& \dot{y}=-y(k y+l x)-(p y+q x) T_{2} \\
& -(2 a x+b y+d)\left[-q x^{2}+(n-p) x y+m y^{2}\right]
\end{aligned}
$$

where $T_{2}=a x^{2}+b x y+c y^{2}+d x+e y+1$. 


$$
\begin{aligned}
C R_{17}^{(12)}: \quad \dot{x}= & k\left[(x-a y+a+2)\left(2 \eta-3 \eta_{y}+3 x^{2}+6 x+6\right)-3 \eta \eta_{y}\right. \\
& \left.-9 x^{2} \eta_{y}+9\left(2 a x^{2}+(2 a-1) x+2 a\right)\right] \\
\dot{y}= & 3 k y\left[(x-a y+a+2)(-3 x+y+2)+3\left(x^{2}+x-2\right)\right]
\end{aligned}
$$

where $\eta=x y-a y^{2}+2 x+2(1+a) y+1-a$. (In Theorem 1 of [8] there is a mistake in the formula for $\eta$.)

Darboux centers. The vector field is of the form

$$
V=\frac{1}{Z} \sum_{i=1}^{r} a_{i} X_{f_{i}} \prod_{j \neq i} f_{j}
$$

where $Z(x, y), f_{i}(x, y)$ are polynomials and $X_{f}$ denotes the Hamiltonian vector field. The function $H=\prod f_{i}^{a_{i}}$ is a first integral for $V$. We present the list of first integrals and the polynomials $Z$.

$$
\begin{aligned}
C D_{1}^{(8)}: H= & x^{\alpha} y^{\beta}(x+y-1)^{\gamma}(a x+b y+1) ; Z=1 \\
C D_{2}^{(8)}: H= & x^{\alpha} y^{\beta}\left(x^{2}+a x y+y^{2}+b x+c y+1\right) ; Z=1 \\
C D_{3}^{(7)}: H= & x^{\alpha}\left(x^{2} y+x y^{2}+a y^{3}+b x y+c y^{2}+d x+e y+1\right) ; Z=1 \\
C D_{4}^{(8)}: H= & (1+x y)^{\alpha}\left(x^{2}+a x y+b y^{2}+c x+d y+1\right) ; Z=1 \\
C D_{5}^{(6)}: H= & x y\left(x^{2}+a y^{2}\right)+x^{3}+b y^{3}+c x^{2} \\
& +d x y+e y^{2}+f x+g y ; Z=1 \\
C D_{6}^{(9)}: H= & \frac{\left(x^{2}+y\right)^{3}}{\left(x^{3}+a x y+b y^{2}+c x+d y+1\right)^{2}} ; Z=1 \\
C D_{7}^{(9)}: H= & \frac{\left(x^{2}+y\right)^{5}}{\left(x^{5}+5 x^{3} y / 2+15 x y^{2} / 8+a x y+b y^{2}+c x+d y+1\right)^{2}} ; Z=1 \\
C D_{8}^{(9)}: H= & \frac{\left(x^{3}+x y+a y+1\right)^{4}}{\left(x^{4}+4 x^{2} y / 3+4 a x y / 3+2 y^{2} / 9+b x+c y+d\right)^{3}} ; Z=1 \\
C D_{9}^{(11)}: H= & \frac{\left(x^{3}+y\right)^{5}}{\left(x^{5}+5 x^{2} y / 3+a x+b y+1\right)^{3}} ; Z=1 \\
C D_{10}^{(11)}: H= & \frac{\left(x^{3}+y\right)^{7}}{\left(x^{7}+7 x^{4} y / 3+14 x y^{2} / 9+a x+b y+1\right)^{3}} ; Z=1 \\
C D_{11}^{(12)}: H= & \frac{\left(x^{4}+x^{2}+y\right)^{5}}{\left(x^{5}+5 x^{3} / 4+5 x y / 4+5 x / 32+a\right)^{4}} ; Z=1 \\
C D_{12}^{(12)}: H= & \frac{\left(x^{4}+x y+1\right)^{3}}{\left(x^{6}+3 x^{3} y / 2+3 x^{2} / 2+3 y^{2} / 8+a\right)^{2}} ; Z=1 \\
C D_{13}^{(10)}: H= & \frac{x^{2-2 \beta}\left(x^{2}+y\right)^{\beta}}{x^{2}+a x+b y+1} ; Z=1 \\
C D_{14}^{(10)}: H= & \frac{x^{3-2 \beta}\left(x^{2}+y\right)^{\beta}}{x^{3}+\beta x y+a x+b y+1} ; Z=1
\end{aligned}
$$




$$
\begin{aligned}
& C D_{15}^{(11)}: H=\frac{x^{3-3 \beta}\left(x^{3}+x^{2}+y\right)^{\beta}}{x^{3}+\beta x^{2}+\beta(\beta-1) x / 2+\beta y+a} ; Z=1 \\
& C D_{16}^{(11)}: H=\frac{x^{4-3 \beta}\left(x^{3}+x^{2}+y\right)^{\beta}}{x^{4}+\beta x^{3}+\left(\begin{array}{c}
\beta \\
2
\end{array}\right) x^{2}+\beta x y+\left(\begin{array}{c}
\beta \\
3
\end{array}\right) x+\beta(\beta-1) y+a} ; Z=1 \\
& C D_{17}^{(11)}: H=\frac{\left(x^{2}+y\right)^{3 / 2-\beta}\left(x^{2}+a y+1\right)^{\beta}}{x^{3}+(3 / 2+(a-1) \beta) x y+\beta x+b} ; Z=1 \\
& \text { where } 4 \beta(\beta-1) a^{2}+4 \beta(3-2 \beta) a+(3-2 \beta)(1-2 \beta)=0 \text {. } \\
& C D_{18}^{(10)}: H=\frac{x^{2-\beta-2 \gamma}(x+1)^{\beta}\left(x^{2}+y\right)^{\gamma}}{x^{2}+\beta x+\gamma y+a} ; Z=1 \\
& C D_{19}^{(10)}: H=\frac{(1+x y)^{\alpha}}{1+\alpha x y+x^{2}(a x+b y+1)} ; Z=x \\
& C D_{20}^{(10)}: H=\frac{(1+x)^{\alpha}(1+x y)^{\beta}}{1+\alpha x+\beta x y+a x^{2}} ; Z=x \\
& C D_{21}^{(10)}: H=\frac{(1+x y)^{\alpha}}{1+\alpha x y+\alpha(\alpha-1) x^{2} y^{2} / 2+x^{3}(a x+b y+1)} ; Z=x^{2} \\
& C D_{22}^{(11)}: H=\frac{\left(1+x+x^{2} y\right)^{\alpha}}{1+\alpha x+\alpha(\alpha-1) x^{2} / 2+\alpha x^{2} y+b x^{3}} ; Z=x^{2} \\
& C D_{23}^{(11)}: H=\frac{\left(1+x+x^{2} y\right)^{\alpha}}{1+\alpha x+\left(\begin{array}{l}
\alpha \\
2
\end{array}\right) x^{2}+\left(\begin{array}{l}
\alpha \\
3
\end{array}\right) x^{3}+\alpha x^{2} y+2\left(\begin{array}{l}
\alpha \\
2
\end{array}\right) x^{3} y+b x^{4}} ; Z=x^{3} \\
& C D_{24}^{(12)}: H=\frac{\left(1+x^{2}+x^{3} y\right)^{\alpha}}{1+\alpha x^{2}+\alpha(\alpha-1) x^{4} / 2+\alpha x^{3} y} ; Z=x^{4} \\
& C D_{25}^{(11)}: H=\frac{y^{3-\alpha}\left(y+x+x^{2}\right)^{\alpha}}{y^{3}+\alpha x y^{2}+\left(\begin{array}{l}
\alpha \\
2
\end{array}\right) x^{2} y+\left(\begin{array}{l}
\alpha \\
3
\end{array}\right) x^{3}+\alpha x^{2} y^{2}+2\left(\begin{array}{c}
\alpha \\
2
\end{array}\right) x^{3} y+a x^{4}} \text {; } \\
& Z=x^{3} \\
& C D_{26}^{(11)}: H=\frac{y^{1-\alpha}\left(y+x y+x^{2}\right)^{\alpha}}{y+\alpha x y+\alpha x^{2}+\alpha(\alpha-1) x^{2} y / 2+a x^{3}} ; Z=x^{2} \\
& C D_{27}^{(11)}: H=\frac{y^{1-\alpha}\left(y+x y+x^{2}\right)^{\alpha}}{y+\alpha x y+\alpha x^{2}+\left(\begin{array}{c}
\alpha \\
2
\end{array}\right) x^{2} y+2\left(\begin{array}{c}
\alpha \\
2
\end{array}\right) x^{3} / 2+\left(\begin{array}{c}
\alpha \\
3
\end{array}\right) x^{3} y+a x^{4}} ; Z=x^{3} \\
& C D_{28}^{(10)}: H=\frac{y^{1-\alpha}\left(y+x+x y+a x^{2}\right)^{\alpha}}{y+\alpha x+\alpha x y+b x^{2}} ; Z=x \\
& C D_{29}^{(12)}: H=\frac{\left(1+x y+x^{3}\right)^{4}}{\left(x^{4}+4 x^{2} y / 3+4 b x y / 3+b\right)^{3}} ; Z=x \\
& C D_{30}^{(11)}: H=\frac{\left(x y^{2}+x+a y+1\right)^{3}}{x\left(x y^{3}+3 x y / 2+3 a y^{2} / 2+3 y / 2+b\right)^{2}} ; Z=1
\end{aligned}
$$




$$
\begin{aligned}
& C D_{31}^{(12)}: H=\frac{\left(x y^{2}+x+1\right)^{5}}{x^{3}\left(x y^{5}+5 x y^{3} / 2+5 y^{3} / 2+15 x y / 8+15 y / 4+a\right)^{2}} ; Z=1 \\
& C D_{32}^{(12)}: H=\frac{\left[(1+x y)^{2}+x^{3}(1+a x)\right]^{3}}{\left[(1+x y)^{3}+3(1+x y) x^{3}(1+a x) / 2+3 x^{6} / 8\right]^{2}} ; Z=x^{6} \\
& C D_{33}^{(10)}: H=\frac{(1+x y)^{1-\beta}\left(1+x+a x y+b x^{2}\right)^{\beta}}{1+\beta x+(1-\beta+a \beta) x y+\left((\beta(\beta-1) / 2+b \beta) x^{2}\right.} ; Z=x^{2} \\
& C D_{34}^{(10)}: H=\frac{\left(y+x^{2}\right)^{1-\beta}\left(y+x+a x^{2}\right)^{\beta}}{y+\beta x+b x^{2}} ; Z=x \\
& C D_{35}^{(10)}: H=\frac{y^{3-\beta}(y+x)^{\beta}}{y^{3}+\beta x y^{2}+\left(\begin{array}{c}
\beta \\
2
\end{array}\right) x^{2} y+\left(\begin{array}{c}
\beta \\
3
\end{array}\right) x^{3}+x^{4}(a x+b y+1)} ; Z=x^{3}
\end{aligned}
$$

\section{REFERENCES}

[1] S. S. Abhyankar And T. T. Moch, Embeddings of the line in the plane, J. Reine Angew. Math. 276 (1975), 148-166.

[2] M. Berthier, D. Cerveau et A. Lins-Neto, Sur les feuilletages analytiques réels et le problème du centre, J. Differential Equations 131 (1996), 244-266.

[3] J. Chavarriga, A class of integrable polynomial vector fields, Appl. Math. (Warsaw) 23 (1995), 339-350.

[4] C. Christopher, Private communication (1994).

[5] A. G. Khovanskir, Topological obstructions to the representability of functions by quadratures, J. Dynam. Control Systems 1 (1995), 91-123.

[6] M. F. Singer, Liouvillian first integrals of differential equations, Trans. Amer. Math. Soc. 333 (1992), 673-688.

[7] J. Sokulski, The beginning of classification of Darboux integrals for cubic systems with center, Differential Integral Equations (to appear).

[8] H. ŻOŁĄDEK, The classification of reversible cubic systems with center, Topol. Methods Nonlinear Anal. 4 (1994), 79-136.

HENRYK ŻOŁĄDEK

Institute of Mathematics

University of Warsaw

Banacha 2

02-097 Warszawa, POLAND

and

Institute of Mathematics

Polish Academy of Sciences

Śniadeckich 8

00-950 Warszawa, POLAND

E-mail address: zoladek@mimuw.edu.pl 